\title{
Incidence of venous thromboembolism in patients with non-hematological cancer admitted for COVID-19 at a third-level hospital in Madrid
}

\author{
Diana Paredes-Ruiz ${ }^{1}$ (1) - Covadonga Gómez-Cuervo ${ }^{1}$. Carlos Gómez-Martín ${ }^{2}$ - Ángel Sánchez-Guerrero ${ }^{3}$. \\ Jesús González-Olmedo ${ }^{1}$ · Flora López-López ${ }^{2}$ - Mateo Bover-Larroya ${ }^{2} \cdot$ Ramón Yarza-Barrio $^{2}$. \\ Diego Dimas Jara-Casas ${ }^{2} \cdot$ Alicia Castelo-Laureiro $^{2} \cdot$ Yolanda Revilla-Ostolaza $^{4} \cdot$ Luis Paz-Ares $^{2}$. \\ Carlos Lumbreras-Bermejo ${ }^{1}$. Carmen Díaz-Pedroche ${ }^{1}$
}

Accepted: 13 April 2021 / Published online: 22 April 2021

( ) The Author(s), under exclusive licence to Springer Science+Business Media, LLC, part of Springer Nature 2021

\begin{abstract}
An increased risk of venous thromboembolism (VTE) in hospitalized patients with COVID-19 has been reported. We aimed to describe the incidence rate of VTE on patients with non-hematological cancer who required hospitalization due to COVID-19 at our center. In this prospective study, non-hematological cancer patients hospitalized for confirmed COVID19 at our institution from 1st March to 30th April 2020, were evaluated daily for VTE complications during their hospital stay, and after discharge until 30th June 2020. Furthermore, Doppler ultrasound of lower limbs was routinely performed in asymptomatic patients based on D-dimer levels and current active cancer therapy. The primary outcome of this study was the cumulative incidence of VTE. Secondary outcomes were the cumulative incidence of bleeding and mortality. A total of 58 hospitalized non-hematological cancer patients and confirmed COVID-19 were identified. Median follow-up since initial symptoms of COVID-19 was 91 days (IQR 19-104). Pulmonary embolism was diagnosed in three (5\%) patients. Symptomatic catheter-related deep vein thrombosis (DVT) was observed in one patient. Doppler ultrasound of lower limbs was done in 11 asymptomatic patients, showing distal DVT in two of them (18\%). The cumulative incidence of VTE on day 14 after admission was $10 \%$, without new VTE events after hospital discharge and up to 90 days follow-up. No bleeding complication was observed. Seventeen patients (29\%) died in the first 14 days after COVID-19 diagnosis. Four patients died after discharge due to malignancy progression. The cumulative incidence of VTE in non-hematological cancer patients under active treatment was $10 \%$ at day 14 after admission, with no further new events in the following 12 weeks.
\end{abstract}

Keywords Cancer $\cdot$ COVID-19 $\cdot$ Deep vein thrombosis $\cdot$ Pulmonary embolism $\cdot$ Venous thromboembolism

\section{Abbreviations \\ VTE Venous thromboembolism \\ DVT Deep vein thrombosis \\ CTPA Computed tomography pulmonary angiogram}

Diana Paredes-Ruiz

diana.paredes@salud.madrid.org

1 Department of Internal Medicine, Hospital Universitario 12 de Octubre, Avenida Cordoba S/N 28041, Madrid, Spain

2 Department of Medical Oncology, Hospital Universitario 12 de Octubre, Madrid, Spain

3 Department of Vascular Radiology, Hospital Universitario 12 de Octubre, Madrid, Spain

4 Department of Radiology, Hospital Universitario 12 de Octubre, Madrid, Spain
PE Pulmonary embolism

LMWH Low-molecular-weight heparin

GFR Glomerular filtration rate

RT-PCR Real time-polymerase chain reaction

LDH Lactate-dehydrogenase

CRP C-reactive protein

ARDS Acute respiratory distress syndrome

$\mathrm{PaFi} \quad$ Arterial oxygen pressure/oxygen inspiration fraction rate $\%$

IQR Interquartile range

ICU Intensive care unit 


\section{Highlights}

- Thrombosis risk in cancer patients with COVID-19 has scarcely been studied.

- The incidence of VTE in non-hematological cancer patients hospitalized for COVID-19 could be similar to that of hospitalized cancer patients without COVID-19.

- Standard dose thromboprophylaxis could prevent VTE without increasing the risk of bleeding.

\section{Introduction}

The novel SARS-CoV-2 causes an infection ranging from asymptomatic or mild symptoms to severe pneumonia with systemic inflammatory response and activation of clotting leading to thrombotic phenomena [1-3].

The prothrombotic state in COVID-19 may explain the increased risk of venous thromboembolism (VTE) reported in these patients, especially for those in intensive care units (ICU) [1,4-9]. Moreover, immobilization can also contribute to thrombosis development regardless of the severity of the disease, as COVID-19 patients may have constitutional symptoms and must remain in isolation.

Cancer patients with acute medical illnesses who require hospital admission are at higher VTE risk during the hospital stay and for up to 90 days post-discharge compared to noncancer patients [10-15]. Additionally, cancer patients are at higher risk of bleeding during hospital stay [16]. Thrombosis risk in cancer patients with COVID-19 has scarcely been studied [7, 17-20], but recent observations have suggested a similar risk of thrombosis and bleeding complications comparing with non-cancer patients [18].

Our main objective was to describe the cumulative incidence of VTE in patients with non-hematological cancer hospitalized for COVID-19. Secondary objectives were to evaluate the cumulative incidence of bleeding and a possible relationship between VTE and mortality.

\section{Methods}

\section{Study design and patients}

A prospective observational study was developed at the Oncology and Internal Medicine Departments of Hospital Universitario 12 de Octubre, Madrid. From 1st March to 30th April 2020, we enrolled all patients with confirmed COVID-19 and non-hematological active cancer. Patients younger than 18 years, and those without microbiological confirmed COVID-19 infection were excluded. Active search for symptoms and signs of VTE was performed daily by the clinical evaluation during hospitalization. Computed tomography pulmonary angiogram (CTPA) and/or Doppler ultrasound were performed whenever pulmonary embolism (PE) or deep vein thrombosis (DVT) were clinically suspected. Doppler ultrasound of lower limbs was performed in otherwise asymptomatic patients if one or more of the following conditions were present: previous VTE disease, active cancer treatment in the past month, or D-dimer levels higher than $1000 \mathrm{ng} / \mathrm{ml}$. Bedside color Doppler ultrasound with compression at $2 \mathrm{~cm}$-interval of the deep veins from the inguinal ligament to the ankle (including posterior tibial and peroneal veins in the calf), right and left common femoral vein spectral Doppler waveforms, popliteal spectral Doppler, and color Doppler images were performed. Patients on anticoagulation with low-molecular-weight heparin (LMWH) before admission continued treatment without modifications while those on oral anticoagulants were changed to LMWH (enoxaparin or tinzaparin weightadjusted, with a 50\% reduction for glomerular filtration rate (GFR) $<30$ or $<20 \mathrm{ml} / \mathrm{min}$, respectively). All patients received prophylactic anticoagulation since hospital admission according to patient weight, kidney function, and platelet count (enoxaparin $40 \mathrm{mg} /$ day if GFR $>30 \mathrm{ml} / \mathrm{min}$ and platelet count of at least $50,000 / \mu \mathrm{L}$; enoxaparin $20 \mathrm{mg} /$ day if GFR $<30 \mathrm{ml} / \mathrm{min}$; enoxaparin $60 \mathrm{mg} /$ day for bodyweight $>80 \mathrm{~kg}$ ). Patients admitted from 1st April to 30th May also maintained prophylactic anticoagulation for the next four weeks following hospital discharge. Patients were followed from discharge to 30th June 2020. Tumor evaluation was performed during de study period according to the oncologist's criteria.

We selected a control group of non-oncological patients. The control group was adjusted by age and time of presentation. Nevertheless, we rejected the comparison because the presence of comorbidities, management, screening strategy and follow-up differed widely between the two groups. Flowchart is shown in the supplementary material.

\section{Variables and definitions}

Active cancer was defined as that diagnosed or under treatment within the previous 6 months, or that on progressive disease or with metastatic spread. Immobilization was considered as total bed rest with bathroom privileges for three or more days. A microbiological confirmed COVID-19 case was defined as that with SARS-CoV-2 real time-polymerase chain reaction (RT-PCR) positive test in nasopharyngeal smear or sputum sample. Risk of immediate complication was defined as older than 65 years, cardiovascular or pulmonary disease, grade 2 or more neutropenia, or expected in the next seven days, presence of bilateral lung infiltrates, 
or the presence of high-risk laboratory markers, such as lactate-dehydrogenase $(\mathrm{LDH})>250 \mathrm{UI} / \mathrm{ml}$, C-reactive protein $(\mathrm{CRP})>5 \mathrm{mg} / \mathrm{dl}$, D-dimers $>2000 \mathrm{ng} / \mathrm{ml}$ or lymphopenia $<1000$ cells $/ \mathrm{ml}$. D-dimer levels were determined by HemoSIL D Dimer HS 500 method. The D-dimer higher value was recorded during the hospitalization or at the time of the thrombotic event in case of VTE occurrence. Recent bleeding was defined as any major bleeding within 30 days before admission. PE was considered whenever thrombosis was diagnosed by CTPA or CT. DVT was assessed by Doppler ultrasound examination. Major bleeding was defined according to the International Society of Thrombosis and Haemostasis description. Acute respiratory distress syndrome (ARDS) was considered if one of the following were present: respiratory rate $>30$ breaths per minute, oxygen saturation $<93 \%$, or arterial oxygen pressure/oxygen inspiration fraction rate $\%(\mathrm{PaFi})<300$. Early death was considered when occurring in the first 14 days after admission.

\section{Statistical analysis}

Qualitative variables are presented by the frequency distribution and percentages. Quantitative variables are summarized by their median and the interquartile range (IQR). The association between qualitative variables was studied with the Chi-squared test and the Fisher Exact test, when appropriate. For quantitative variables, the t-test or the Mann-Whitney U test was performed. We used Kaplan Maier and Cox regression to determine survival curves and hazard rates. The cumulative incidences of thrombosis or bleeding complications were estimated considering death as a competing risk. A two-sided $\mathrm{p}<0.05$ was considered statistically significant. SPSS software (version 25, SPSS Inc., Chicago, Illinois) and STATA 15.1 were used for statistical analysis.

\section{Ethics}

This study was reviewed and approved by the Local Research Ethics Committee and following the Declaration of Helsinki, Good Clinical Practice, and local legal requirements. Due to the observational nature of the study and the pandemic situation, patients were included after verbal informed consent was obtained.

\section{Results}

Fifty-eight non-hematological cancer patients with confirmed COVID-19 were included. The median age was 67 (IQR 62-73) years and 52\% were males. The most frequent primary tumor was lung cancer (28\%) (Table 1). The median follow-up since COVID-19 symptoms onset was
Table 1 Clinical characteristics at baseline and cancer description

\begin{tabular}{|c|c|}
\hline Baseline characteristics & $\mathrm{N}=58$ \\
\hline Median age, years (IQR) & $67(62-73)$ \\
\hline Sex (males), n (\%) & $30(52)$ \\
\hline \multicolumn{2}{|l|}{ Race, n (\%) } \\
\hline Caucasian & $51(88)$ \\
\hline Hispanic & $5(9)$ \\
\hline Other* & $2(3)$ \\
\hline \multicolumn{2}{|l|}{ Performance status, n (\%) } \\
\hline 0 & $17(29)$ \\
\hline 1 & $34(59)$ \\
\hline 2 & $6(10)$ \\
\hline 3 & $1(2)$ \\
\hline \multicolumn{2}{|l|}{ Smoker, n (\%) } \\
\hline Former & $28(48)$ \\
\hline No & $27(47)$ \\
\hline Active & $3(5)$ \\
\hline Hypertension, n (\%) & $30(52)$ \\
\hline Cardiovascular disease, $\mathrm{n}(\%)$ & $10(17)$ \\
\hline Pulmonary disease, $\mathrm{n}(\%)$ & $12(21)$ \\
\hline Kidney disease (GFR $<30 \mathrm{ml} / \mathrm{min} / \mathrm{m} 2), \mathrm{n}(\%)$ & $1(2)$ \\
\hline Obesity, n (\%) & $1(2)$ \\
\hline Previous VTE, n (\%) & $10(17)$ \\
\hline Bleeding history, n (\%) & $5(9)$ \\
\hline Prior month bleeding, n (\%) & $3(5)$ \\
\hline Antiplatelet use, n (\%) & $9(16)$ \\
\hline \multicolumn{2}{|l|}{ Previous anticoagulation, $\mathrm{n}(\%)$} \\
\hline Previous anticoagulant dose & $11(19)$ \\
\hline Prophylactic & $1(2)$ \\
\hline Therapeutic & $10(17)$ \\
\hline \multicolumn{2}{|l|}{ Previous anticoagulation drug } \\
\hline DOAC & $3(5)$ \\
\hline LMWH & $8(14)$ \\
\hline \multicolumn{2}{|l|}{ Previous anticoagulation indication } \\
\hline VTE & $7(12)$ \\
\hline Atrial fibrillation & $4(7)$ \\
\hline Cancer characteristics & $\mathrm{N}=58$ \\
\hline \multicolumn{2}{|l|}{ Primary tumor, $\mathrm{n}(\%)$} \\
\hline Lung & $16(28)$ \\
\hline Breast & $10(17)$ \\
\hline Colorectal & $8(14)$ \\
\hline Prostate & $5(9)$ \\
\hline Urothelial & $3(5)$ \\
\hline Head and neck & $3(5)$ \\
\hline Pancreatic and hepatobiliary & $4(3)$ \\
\hline Ovarian & $2(3)$ \\
\hline Other $^{\dagger}$ & $7(12)$ \\
\hline Metastatic disease, n (\%) & $48(83)$ \\
\hline Pulmonary tumor involvement, n (\%) & $22(38)$ \\
\hline \multicolumn{2}{|l|}{ Treatment, n (\%) } \\
\hline Chemotherapy alone & $26(45)$ \\
\hline Immunotherapy alone & $9(16)$ \\
\hline
\end{tabular}


Table 1 (continued)

\begin{tabular}{ll}
\hline Baseline characteristics & $\mathrm{N}=58$ \\
\hline Chemotherapy and immunotherapy & $5(9)$ \\
Hormonotherapy & $9(16)$ \\
Other & $6(10)$ \\
None & $3(5)$ \\
Antiangiogenic therapy, n $(\%)$ & $8(14)$ \\
\hline
\end{tabular}

$I Q R$ interquartile range, GFR glomerular filtration rate, VTE venous thromboembolism, DOAC direct oral anticoagulant, $L M W H$ lowmolecular-weight heparin

*Other races: Arabian 1, African 1

Other primary tumors: Thyroid 2, unknown origin 1, kidney 1, gastric 1, anal 1, gastrointestinal stromal tumor 1

Other treatments: Tyrosine-kinase inhibitor 4, RET inhibitor 1, radionuclide 1

91 days (IQR 19-104). Fifty-five (95\%) patients presented lung infiltrates (bilateral 83\%, lobar 12\%), and four (7\%) patients had grade 4 neutropenia at admission. Twenty-five (43\%) patients developed ARDS. None of these patients were admitted to the ICU or underwent mechanical ventilation. Seventeen (29\%) patients died in the first 14 days after admission due to ARDS. The cumulative mortality rate was $36 \%$ at the end of the study. Four (10\%) patients died after they had been discharged because of cancer-related issues.

Eleven (19\%) patients were on anticoagulation at admission. Indications for anticoagulation were extended therapy for venous thromboembolism in seven patients and atrial fibrillation in four patients. All patients were under therapeutic doses, except for one who was on prophylactic doses. Two patients started therapeutic anticoagulation upon admission due to a concomitant diagnosis of VTE. The rest of the patients underwent prophylactic anticoagulation at standard doses upon their admittance to the hospital except in one case with persistent grade 4 thrombocytopenia. After hospital discharge, 28 (48\%) patients continue anticoagulation therapy; nine of them (32\%) at therapeutic doses, and $19(68 \%)$ at prophylactic doses to complete an outpatient course of additional four weeks. All patients with new-onset VTE started anticoagulation at therapeutic doses at the time of suspicion or diagnosis of the event. None of the patients received intermediate doses of anticoagulation. Antiplatelet agents for secondary cardiovascular prevention were maintained during hospitalization in those patients who did not receive therapeutic anticoagulation. Hormone therapies were withdrawn at admission and did not resume until clinical recovery after discharge.

Twenty-three (40\%) patients had a chest CT performed during the inpatient care and 32 (78\% of those discharged) once they have been discharged. Three (5\%) patients developed clinically suspected and radiologically confirmed PE. The extension of all three PE was lobar and segmental. We did not observe any incidental PE. One patient had a symptomatic catheter-related DVT. Eleven (19\%) asymptomatic patients were screened by a Doppler ultrasound of lower limbs, showing distal DVT in two (18\%) patients. The median time from the onset of COVID-19 symptoms to VTE diagnosis was 9 days (IQR 2-19). The median time from the hospital admission to VTE diagnosis was 7.5 days (IQR 0-13). All VTE events occurred in the first two weeks after hospitalization. None of the patients suffered a VTE event after discharge, independently of the use or not of prophylaxis in the outpatient setting. The crude cumulative incidence of VTE, including PE and DVT, was $10 \%$ on day 14 from admission, with no differences after two weeks of admission and after hospital
Fig. 1 Cumulative incidence of venous thromboembolism

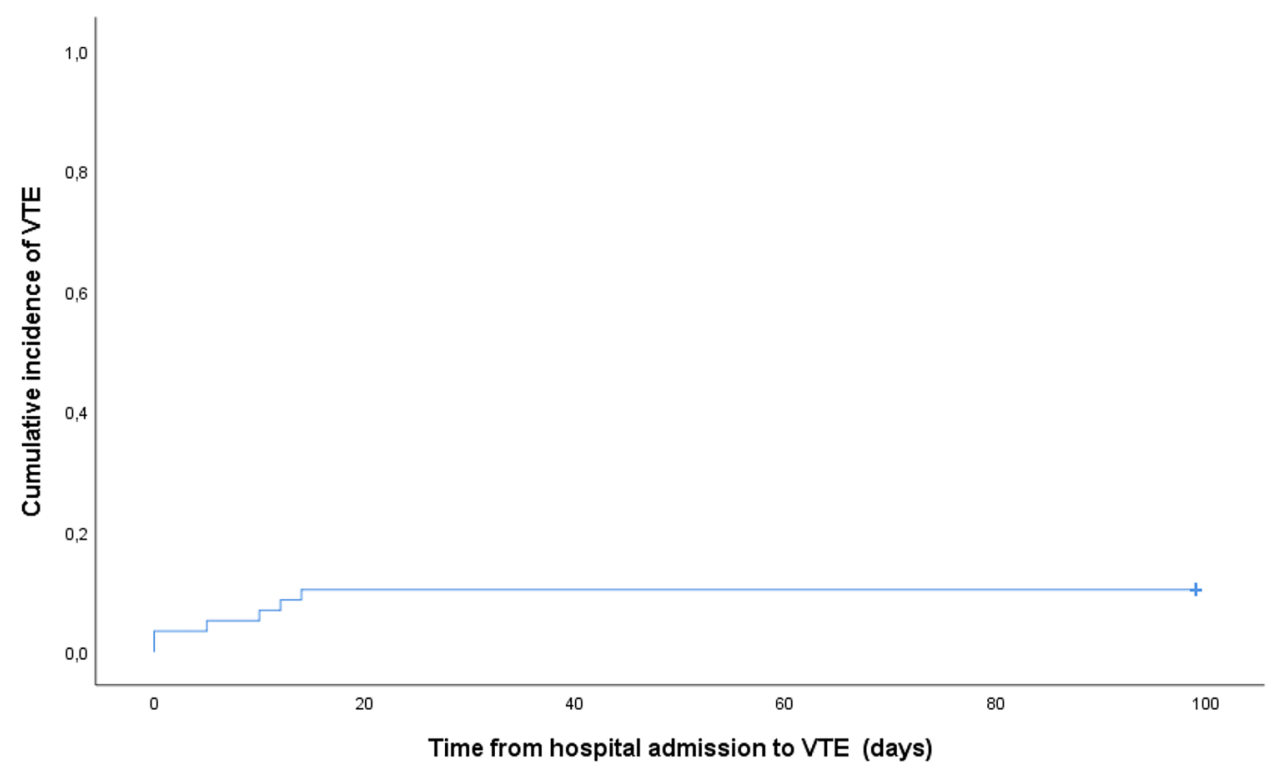


discharge (Fig. 1). Adjustment for competing risk of death could not be performed due to the low number of VTE events. The incidence rate was 5 cases $/ 100$ person-month. None of the patients developed multiple site venous thromboses or arterial thrombotic events.

Patients with VTE were non-significantly younger than those without VTE (median age 63 -IQR 57-66- vs. 68 -IQR 63-74-, p 0.09). Males had a non-significant higher VTE rate (7\% vs. $3 \%$, OR 2 (95\% CI 0.34-11.89, p 0.671). Lung cancer was the most prevalent primary tumor among those with VTE (33\%). All patients with VTE had metastatic spread of their disease and chemotherapy was their main treatment (83\%). D-dimer levels were higher in those with VTE (median $9729 \mathrm{ng} / \mathrm{ml}$-IQR 4120-21,300- vs. median 1610 ng/ml -IQR 695-2620-, p 0.002). We did not observe any association between previous VTE (OR 0.88 (95\% CI $0.79-0.97), p$ 0.577) or the use of anticoagulation before admission (OR 0.84 (95\% CI 0.09-8.01), p 1.) and the occurrence of new VTE events. ARDS and early-mortality rate were higher in those without VTE (44\% vs. $33 \%$ and $31 \%$ vs. $17 \%$, respectively), although no association was observed between ARDS (OR 0.63 (95\% CI 0.11-3.75), p 0.690) or COVID-19 related death (OR 0.45 (95\% CI 0.05-4.17), p 0.660) and the development of VTE. Patients with VTE had a higher mortality rate after discharge due to cancer progression than those without VTE (OR 53 (95\% CI 3.62-761.44), p 0.004); two patients with PE and one patient with catheter-DVT died vs. one death in the group without VTE. The median survival time was longer in patients without VTE (87 days -IQR 17-95-) compared to those with VTE (74 days -IQR 34-88-), p 0.219 (Fig. 2). None of the patients in this study developed bleeding complications related to anticoagulant therapy or their underlying neoplasm. Comparison of patients according to the presence of VTE event is shown in Table 2.

\section{Discussion}

In our cohort, the crude cumulative incidence of both symptomatic and asymptomatic VTE in non-hematological cancer patients with COVID-19 was $10 \%$ at 90 days. We did not observe new events beyond day 14 after admission. Our incidence is lower than those reported in previous studies, which demonstrated VTE incidence rates between 5 to $50 \%$ in hospitalized patients with COVID-19. However, these studies are highly heterogeneous, with large differences in the severity of the disease, comprising ICU and non-ICU patients, screening DVT strategies, anticoagulation protocols, and also including mixed arterial and venous thrombosis [4-9, 17-20]. Besides, few of them included active cancer patients [17-20], and, to our knowledge, this is the first study specifically designed to assess this problem in non-hematological cancer patients. A recent study found a lower risk of thrombosis in cancer patients compared to noncancer patients with COVID-19 (14\% vs. $18 \%$ at 28 days), supporting our results [18]. When we compare our results with other studies analyzing the incidence of hospital-related thrombosis in patients with cancer, the incidence of VTE in our cohort is similar to those reported in non-COVID-19 hospitalized cancer patients. In these studies, the incidence ranges from 2 to $22 \%$, with higher rates reported in prospective trials utilizing routine screening for VTE $[15,21]$.

Anticoagulant prophylaxis reduces the risk of VTE in hospitalized medical patients [10,13]. Some studies have suggested that hospitalized patients with active cancer could
Fig. 2 Overall survival of cancer patients based on their venous thromboembolism (VTE) status

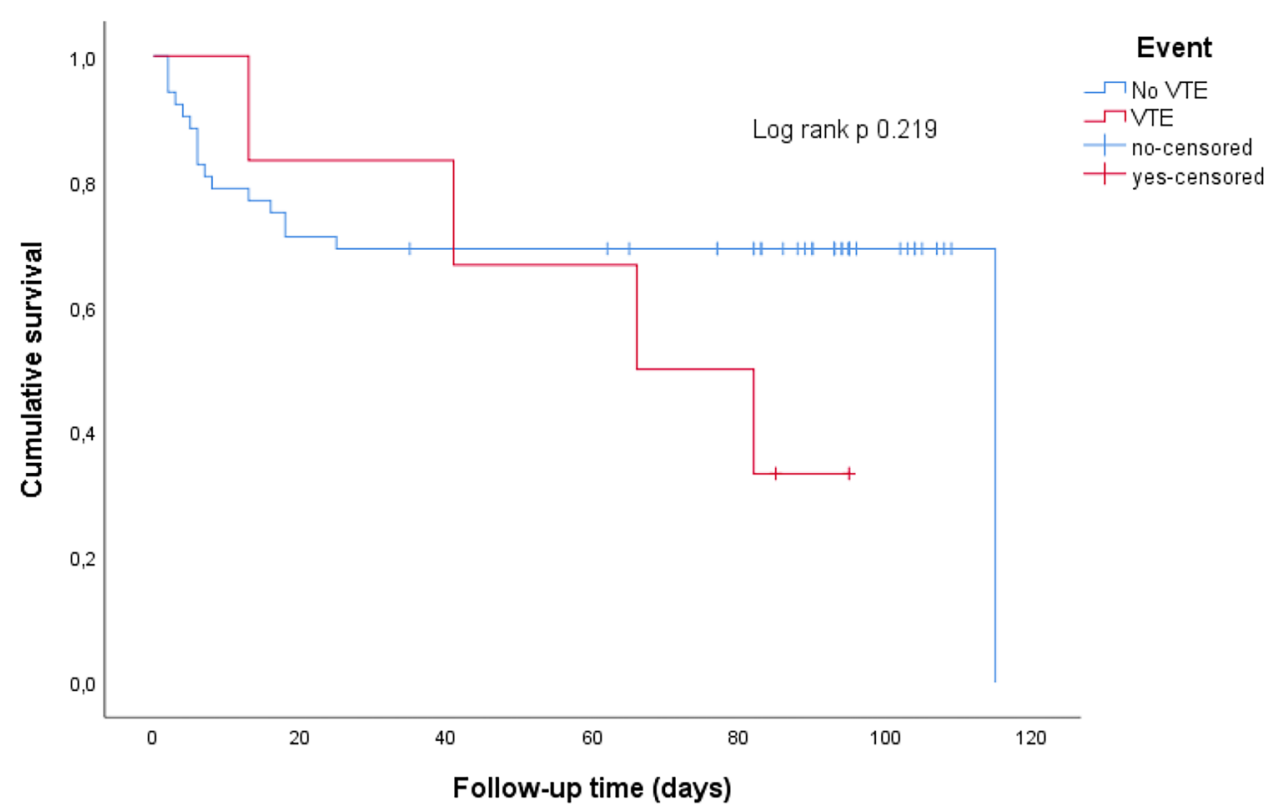


Table 2 Comparison of patients according to the presence of venous thromboembolism

\begin{tabular}{|c|c|c|c|}
\hline Baseline characteristics & Non-VTE $(\mathrm{N}=52)$ & $\operatorname{VTE}(\mathrm{N}=6)$ & $p$-value \\
\hline Median age, years (IQR) & $68(63-74)$ & $63(57-66)$ & 0.090 \\
\hline \multicolumn{4}{|l|}{ Sex } \\
\hline Male, $\mathrm{n}(\%)$ & $26(50)$ & $4(67)$ & 0.671 \\
\hline Female, n $(\%$ & $26(50)$ & $2(33)$ & \\
\hline Previous VTE, n (\%) & $10(19)$ & 0 & 0.577 \\
\hline Previous therapeutic anticoagulation, n (\%) & $10(19)$ & $1(17)$ & 1.000 \\
\hline Cancer characteristics & Non-VTE $(\mathrm{N}=52)$ & $\operatorname{VTE}(\mathrm{N}=6)$ & \\
\hline \multicolumn{4}{|l|}{ Primary tumor, $\mathrm{n}(\%)$} \\
\hline Lung & $14(27)$ & $2(33)$ & \\
\hline Breast & $9(17)$ & $1(17)$ & \\
\hline Colorectal & $8(15)$ & 0 & \\
\hline Prostate & $4(8)$ & $1(17)$ & \\
\hline Urothelial & $3(6)$ & 0 & \\
\hline Head and neck & $3(6)$ & 0 & \\
\hline Pancreatic & $1(2)$ & $1(17)$ & \\
\hline Ovarian & $2(3,8)$ & 0 & \\
\hline Hepatobiliary & $2(4)$ & 0 & \\
\hline Metastatic disease, n (\%) & $42(81)$ & $6(100)$ & 0.577 \\
\hline Pulmonary involvement, $\mathrm{n}(\%)$ & $19(37)$ & $3(50)$ & 0.664 \\
\hline \multicolumn{4}{|l|}{ Treatment, n (\%) } \\
\hline Chemotherapy alone & $22(42)$ & $4(67)$ & \\
\hline Immunotherapy alone & $9(17)$ & 0 & \\
\hline Chemotherapy and immunotherapy & $4(8)$ & $1(17)$ & \\
\hline Hormonotherapy & $9(17)$ & 0 & \\
\hline RET inhibitor & 0 & $1(17)$ & \\
\hline Chemotherapy vs. others & $26(50)$ & $5(83)$ & 0.201 \\
\hline COVID-19 outcomes & Non-VTE $(\mathrm{N}=52)$ & $\operatorname{VTE}(\mathrm{N}=6)$ & \\
\hline Median D-dimer, ng/ml (IQR) & $1610(695-2620)$ & $9729(4120-21,300)$ & 0.002 \\
\hline Distress, n (\%) & $23(44)$ & $2(33)$ & 0.690 \\
\hline Mortality & Non-VTE $(\mathrm{N}=52)$ & $\operatorname{VTE}(\mathrm{N}=6)$ & \\
\hline Death, n (\%) & $17(33)$ & $4(67)$ & 0.176 \\
\hline COVID-19 related death, $\mathrm{n}(\%)$ & $16(31)$ & $1(17)$ & 0.660 \\
\hline Malignancy related death, n (\%) & $1(3)$ & $3(60)$ & 0.004 \\
\hline Bleeding, n (\%) & 0 & 0 & \\
\hline
\end{tabular}

$I Q R$ interquartile range, VTE venous thromboembolism

require higher anticoagulant doses than non-cancer patients to find a significant reduction of VTE [21]. Nevertheless, in the absence of randomized studies in this population, and considering a risk reduction of VTE of $50 \%$ in some studies [10], guidelines recommend pharmacologic thromboprophylaxis for hospitalized patients with cancer and reduced mobility at a similar dose as for non-cancer patients unless any contraindication exists [22]. In our cohort, standard thromboprophylaxis was used according to our hospital protocol in all patients who were not receiving therapeutic anticoagulation for other indications. The wide use of anticoagulation could influence the lower rates of VTE in our cohort.

We did not observe any proximal DVT in the screening strategy achieved in those with higher thrombosis risk. None of the two patients with asymptomatic distal DVT died at follow-up. Although the sample size limits interpretation, we observed that the increased thrombus load in the pulmonary circulation showed a worse prognosis than those with distal DVT. 
None of the patients had major bleeding despite up to $20 \%$ of them were on therapeutic anticoagulation and nearly $50 \%$ of the subjects continued anticoagulation therapy after hospital discharge (68\% at prophylactic doses for additional four weeks). As we have previously mentioned, Patell et al. [18] recorded a slightly higher incidence of VTE in a cohort of cancer patients with COVID-19, and also reported a higher incidence of bleeding (20\%). However, patients included in that study had a more severe COVID-19 disease (up to $40 \%$ required invasive ventilation), and the anticoagulants use during hospitalization differed from our cohort (7\% did not receive any anticoagulant, while about $60 \%$ were on high doses of thromboprophylaxis or therapeutic anticoagulation). Our results suggest that standard thromboprophylaxis could be a reasonable option for non-hematological cancer patients admitted for COVID-19 in a non-ICU setting.

The mortality in our cohort was high, mainly due to COVID-19. We did not find an association between VTE and mortality. However, 90-days survival rates showed a non-significant higher malignancy-related mortality rate in patients who developed VTE. This fact highlights the role of VTE as a manifestation of advanced disease [23, 24].

Our study had some limitations. First, the main concern is the lack of a control group owing to the difficulty in its selection. Only an indirect comparison with available literature data was performed. Second, the small size of the sample limits the statistical power to find relevant differences between subgroups (symptomatic vs. asymptomatic, extended prophylactic anticoagulation vs. non-extended prophylactic anticoagulation). Third, DVT screening strategy and extended prophylactic anticoagulation were implemented during the first weeks of the pandemics as concern on VTE emerged. Moreover, $17 \%$ of patients were already on anticoagulation treatment at therapeutic doses at admission. These different anticoagulation approaches make it difficult to interpret the benefit of prophylactic doses of anticoagulation. Finally, none of the patients with severe ARDS underwent mechanical ventilation due to the short life expectancy related to cancer disease, and scarce medical resources during the first weeks of pandemics in Spain. The high early mortality rate could result in a competitive risk for developing thrombosis. Thus, the incidence of thrombosis can not be compared to that of cancer and non-cancer population in the ICU setting.

\section{Conclusion}

Patients with non-hematological cancer hospitalized for COVID-19 showed a cumulative incidence of VTE of $10 \%$ at day 14 after admission, without further new events in the following weeks.
Supplementary Information The online version contains supplementary material available at https://doi.org/10.1007/s11239-021-02448-w.

Acknowledgements The authors wish to thank the patients, their families and all those healthcare workers involved in this study, as well as to all cancer patients and their families suffering this disease.

Author contributions All authors contributed to the organization of care, clinical activity, and diagnostic procedures. Doppler ultrasound was achieved by ASG. The study was designed by DPR and CDP. Data collection was performed by RYB, MBL, FLL, DDJ, JGO, CDP. Analysis and interpretation of data were performed by DPR and CGC. All authors reviewed and contributed to the final approval of the manuscript.

Funding This research received no specific grant from any funding agency in the public, commercial or not-for-profit sectors.

\section{Declarations}

Conflict of interest The authors have no conflicts of interest to disclose.

\section{References}

1. Bikdeli B, Madhavan MV, Jimenez D, Chuich T, Dreyfus I, Driggin E et al (2020) COVID-19 and thrombotic or thromboembolic disease: implications for prevention, antithrombotic therapy, and follow-up: JACC state-of-the-art review. J Am Coll Cardiol 75:2950-2973

2. Moores LK, Tritschler T, Brosnahan S, Carrier M, Collen JF, Doerschug K et al (2020) Prevention, diagnosis, and treatment of VTE in patients with coronavirus disease 2019: CHEST guideline and expert panel report. Chest 158:1143-1163

3. Thachil J, Tang N, Gando S, Falanga A, Cattaneo M, Levi M et al (2020) ISTH interim guidance on recognition and management of coagulopathy in COVID-19. J Thromb Haemost 18:1023-1026

4. Cui S, Chen S, Li X, Liu S, Wang F (2020) Prevalence of venous thromboembolism in patients with severe novel coronavirus pneumonia. J Thromb Haemost 18:1421-1424

5. Klok FA, Kruip MJHA, van der Meer NJM, Arbous MS, Gommers DAMPJ, Kant KM et al (2020) Incidence of thrombotic complications in critically ill ICU patients with COVID-19. Thromb Res 191:145-147

6. Helms J, Tacquard C, Severac F, Leonard-Lorant I, Ohana M, Delabranche X et al (2020) High risk of thrombosis in patients with severe SARS-CoV-2 infection: a multicenter prospective cohort study. Intensive Care Med 46:1089-1098

7. Nahum J, Morichau-Beauchant T, Daviaud F, Echegut P, Fichet J, Maillet JM et al (2020) Venous thrombosis among critically ill patients with coronavirus disease 2019 (COVID-19). JAMA Netw Open 3:e2010478

8. Al-Samkari H, Karp Leaf RS, Dzik WH, Carlson JCT, Fogerty AE, Waheed A et al (2020) COVID-19 and coagulation: bleeding and thrombotic manifestations of SARS-CoV-2 infection. Blood 136:489-500

9. Zhang L, Feng X, Zhang D, Jiang C, Mei H, Wang J et al (2020) Deep vein thrombosis in hospitalized patients with COVID-19 in Wuhan, China: prevalence, risk factors, and outcome. Circulation 142:114-128

10. Alikhan R, Cohen AT, Combe S, Samama MM, Desjardins L, Eldor A et al (2003) Prevention of venous thromboembolism 
in medical patients with enoxaparin: a subgroup analysis of the MEDENOX study. Blood Coagul Fibrinolysis 14:341-346

11. Patell R, Rybicki L, McCrae KR, Khorana AA (2017) Predicting risk of venous thromboembolism in hospitalized cancer patients: utility of a risk assessment tool. Am J Hematol 92:501-507

12. Khorana AA, Francis CW, Culakova E, Fisher RI, Kuderer NM, Lyman GH (2006) Thromboembolism in hospitalized neutropenic cancer patients. J Clin Oncol 24:484-490

13. Cohen AT, Turpie AGG, Leizorovicz A, Olsson CG, Vaitkus PT, Goldhaber SZ (2007) Thromboprophylaxis with dalteparin in medical patients: which patients benefit? Vasc Med 12:123-128

14. Merkow RP, Bilimoria KY, McCarter MD, Cohen ME, Barnett CC, Raval MV et al (2011) Post-discharge venous thromboembolism after cancer surgery: extending the case for extended prophylaxis. Ann Surg 254:131-137

15. Bin Yu Y, Gau JP, Liu CY, Yang MH, Chiang SC, Hsu HC et al (2012) A nation-wide analysis of venous thromboembolism in 497,180 cancer patients with the development and validation of a risk-stratification scoring system. Thromb Haemost 108:225-235

16. Decousus H, Tapson VF, Bergmann JF, Chong BH, Froehlich JB, Kakkar AK et al (2011) Factors at admission associated with bleeding risk in medical patients: findings from the improve investigators. Chest 139:69-79

17. Demelo-Rodríguez P, Cervilla-Muñoz E, Ordieres-Ortega L, Parra-Virto A, Toledano-Macías M, Toledo-Samaniego N et al (2020) Incidence of asymptomatic deep vein thrombosis in patients with COVID-19 pneumonia and elevated D-dimer levels. Thromb Res 192:23-26

18. Patell R, Bogue T, Bindal P, Koshy A, Merrill M, Aird WC et al (2020) Incidence of thrombosis and hemorrhage in hospitalized cancer patients with COVID-19. J Thromb Haemost 18:2349-2357
19. Lodigiani C, Iapichino G, Carenzo L, Cecconi M, Ferrazzi P, Sebastian $\mathrm{T}$ et al (2020) Venous and arterial thromboembolic complications in COVID-19 patients admitted to an academic hospital in Milan, Italy. Thromb Res 191:9-14

20. Santoliquido A, Porfidia A, Nesci A, De Matteis G, Marrone G, Porceddu E et al (2020) Incidence of deep vein thrombosis among non-ICU patients hospitalized for COVID-19 despite pharmacological thromboprophylaxis. J Thromb Haemost 18:2358-2363

21. Levitan N, Dowlati A, Remick SC, Tahsildar HI, Sivinski LD, Beyth R et al (1999) Rates of initial and recurrent thromboembolic disease among patients with malignancy versus those without malignancy: risk analysis using medicare claims data. Medicine (Baltimore) 78(5):285-291

22. Key NS, Khorana AA, Kuderer NM, Bohlke K, Lee AYY, Arcelus JI et al (2019) Venous thromboembolism prophylaxis and treatment in patients with cancer: ASCO clinical practice guideline update. J Clin Oncol 38:496-520

23. Gussoni G, Frasson S, La Regina M, Di Micco P, Monreal M (2013) Three-month mortality rate and clinical predictors in patients with venous thromboembolism and cancer. Findings from the RIETE registry. Thromb Res 131:24-30

24. Fuentes HE, Tafur AJ, Caprini JA, Alatri A, Trujillo-Santos J, Farge-Bancel D et al (2019) Prediction of early mortality in patients with cancer-associated thrombosis in the rIete database. Int Angiol 38:173-184

Publisher's Note Springer Nature remains neutral with regard to jurisdictional claims in published maps and institutional affiliations. 